\title{
Alternative rapeseed based oil fluid for hydraulic systems of tractors
}

\author{
Alexander P. Bychenin*, Oleg S. Volodko, Denis N. Bazhutov \\ Samara State Agrarian University, Samara, 446442, the Russian Federation
}

\begin{abstract}
The paper analyzes the main applications of alternative fuels and lubricants in automotive vehicles, considers the possibility of using vegetable oils as a working fluid for hydraulic systems of agricultural machinery. Based on the laboratory findings, it states the ways to improve a lubrication formula based on rapeseed oil. The formula is proven to be optimal if it includes $88.9 \%$ rapeseed oil $+3.7 \%$ D-11+ $3.2 \% \mathrm{EFO}+4.197 \%$ graphite $+0.003 \% \mathrm{MS}-200 \mathrm{~A}$, which is superior in tribological properties to MGE-46V hydraulic oil, and can be recommended for use in hydraulic systems of agricultural machinery as an alternative to mineral oils.
\end{abstract}

\section{Introduction}

Today, fresh and waste lubricants for automotive vehicles are one of the main environmental pollutants. Oil leaks cause soil infertility for a long time, since petroleum and synthetic lubricating oils have low biodegradability and stay in the topsoil layer for decades. The very fact that, for example, for hydraulic systems of agricultural machinery, the top-up rates fluctuate in the range of 1.9-25 liters for every 1000 motor-hours illustrates the relevance of the problem. In fact, breaching the rules of operation leads to an overconsumed rate of working fluids in the hydraulic system by $2-3$ times $[1,2]$. In addition, mineral and synthetic lubricating oils are toxic.

In the 21 st century, primary mineral, animal and plant resources, as well as secondary resources subject to comprehensive processing and safe disposal, remain priority raw materials exploited for the production of fuel and lubricants. Secondary raw materials imply, for example, waste lubricating oils that have been recycled or regenerated for reuse [3, 4]. Besides, methane and landfill gas produced by decomposing organic waste are a similar resource $[5,6]$. This trend stems from the environmental hazard, as well as a reduction in proven reserves of minerals.

The most technically promising biodegradable resource is vegetable oils that are distinguished by a lower cost compared to synthetic and mineral oils. In addition, oils are more environmentally friendly. They are composed of complete glycerol esters and higher monobasic carboxylic acids (Table 1).

There is a wide range of applications for raw plant materials $[7,8]$. In a number of countries, oilseeds with a defined fatty-acid profile are more increasingly produced, which allows their targeted use for food and technical purposes. The most common commodity crop for lubricants in Europe is rapeseed. In Germany, this crop is cultivated on 2.3 million hectares with an average seed yield of $30 \mathrm{c} /$ ha and a pure oil yield of $32 \%$ [9]. In the Russian Federation, the basic oilseed raw material for technical purposes is such cultivated early-flowering plants as winter rape, flax, camelina, mustard, safflower and soybeans. In addition, spinning crops like cotton, fiber flax, hemp are cultivated for multiple use [10].

Table 1. Composition of vegetable oils, $\%$.

\begin{tabular}{|c|c|c|c|c|}
\hline \multirow{2}{*}{ Acid } & \multicolumn{4}{|c|}{ Oils } \\
\cline { 2 - 5 } & Flaxseed & Rapeseed & Sunflower & Colza \\
\hline Myristic & - & 0.5 & traces & - \\
\hline Palmitic & $6-7$ & 2 & $4-9$ & 3 \\
\hline Stearic & $3-5$ & 1 & $3-6$ & 1 \\
\hline Arachidic & traces & 0.5 & 0.5 & 0.5 \\
\hline Behenic & - & 1 & 0.5 & 1 \\
\hline Lignoceric & traces & 1 & - & - \\
\hline Palmitoleic & - & traces & traces & - \\
\hline Oleic & $20-26$ & 15 & $14-35$ & 30 \\
\hline Gadoleic & - & 7 & - & 4 \\
\hline Erucic & - & 50 & - & 25 \\
\hline Linolic & $14-20$ & 15 & $50-75$ & 18 \\
\hline Linolenic & $51-54$ & 7 & 0.1 & 7 \\
\hline
\end{tabular}

\section{Materials and methods}

\subsection{Object of study}

An object of study is rapeseed oil being the most promising basis for a lubricating formula to constitute a working fluid for hydraulic systems of agricultural machinery. The volume of world production of rapeseed

\footnotetext{
*orresponding author: bap63@mail.ru
} 
oil makes up 8 million tons per year, including 2 million tons in the EEC countries. Therefore, in the face of aggravated environmental issues, this type of raw material is likely to displace up to $50 \%$ of petroleum oils and diesel fuel in the future.

Besides being a raw material, rapeseed as a crop is highly effective for green manure, protection of soil from water and wind erosion, as well as for combating weeds and increasing the green cover of fields.

Rapeseed oil is distinguished by a significant content of palmitic, stearic, oleic and other unsaturated organic acids (Table 1), which are surface-active substances (surfactants) capable of forming a damper film on friction surfaces due to adhesive bonds, which is particularly valuable for frictional couples that prevent the use of high-viscosity lubricants. An example of such mates are friction pairs of gear pumps and hydraulic valves of hydraulic systems. They are lubricated with a working fluid. Organic surfactants found to be present there has a significant effect on friction regimes, thus promoting servovite films.

One of the main constraints on the use of vegetable oils in hydraulic systems of agricultural machinery is their aging, that is, changing physical, chemical and operational properties caused by temperature, duration of operation, abrasive content, as well as oxygen (air) access and water availability. The main physical and chemical properties of rapeseed oil in comparison with other oils are shown in Table 2.

Table 2. Comparison of vegetable and mineral oils.

\begin{tabular}{|c|c|c|c|c|}
\hline Indices & Rapeseed & Flaxseed & Colza & M10G \\
\hline Density, kg/m ${ }^{3}$ & 916 & 928 & 911 & 920 \\
\hline $\begin{array}{c}\text { Viscosity at } \\
100^{\circ} \mathrm{C}, \mathrm{mm}^{2} / \mathrm{s}\end{array}$ & 8,3 & 4 & 5 & 11.2 \\
\hline $\begin{array}{c}\text { Contamination, \% } \\
\text { Dispersant and } \\
\text { stabilizing } \\
\text { properties, points }\end{array}$ & - & 0.01 & 0.01 & 0.01 \\
\hline $\begin{array}{c}\text { Base number, mg } \\
\text { KOH/g }\end{array}$ & 6 & 1.5 & - & 6.05 \\
\hline $\begin{array}{c}\text { Temperature, }{ }^{\circ} \mathrm{C}: \\
\text { flash parameter } \\
\text { pour parameter }\end{array}$ & 282 & 316 & 314 & 205 \\
\hline
\end{tabular}

According to the data, the target vegetable oils are similar in density and contamination, and superior to mineral oils in flash and pour point and, hence, they can serve as their best substitute or additive. Besides, rapeseed oil has an advantage over flaxseed and rapeseed oil in viscosity.

The data suggest that alternative biodegradable lubricants can be suitable as a basis for lubricant formulas used in hydraulic systems of agricultural machinery. However, the use of pure vegetable oils is impractical, since they have less thermal-oxidative stability and wear-reducing value. A way to address this challenge is targeted additization with multiple-purpose compounds.

Thus, there is a pressing issue of adapting raw plant materials for use as working fluids in hydraulic systems of agricultural machinery. It can be solved by creating lubricating compositions based on vegetable oils, in particular, rapeseed oil, doped with an additive package.

\subsection{Experimental method}

These studies were carried out for a comparative evaluation of rapeseed oil with mineral oils in order to set forth guidelines for the selection of a package of additives that improve the properties of rapeseed oil, as well as to determine the optimal concentrations of additives in a blended lubricant based on a multifactorial experiment. Oil samples were examined on a 2070 SMT1 roller wear tester and a MAST-1 four-ball wear tester (Fig. 1).

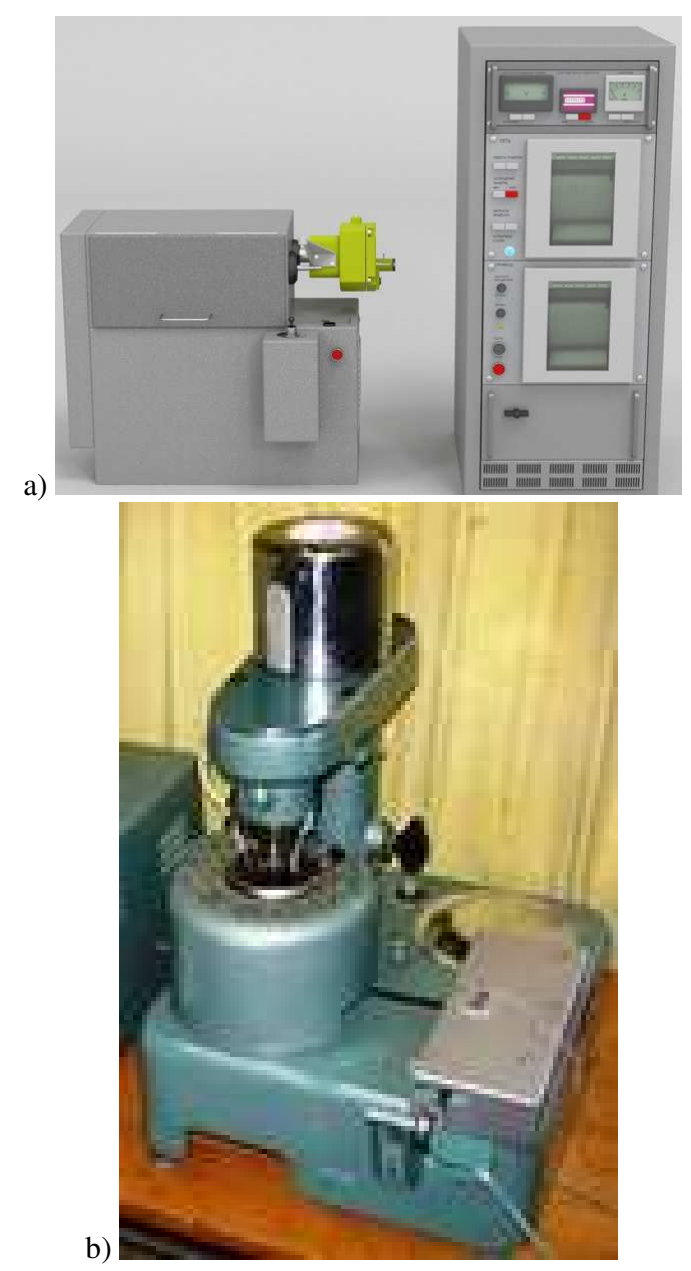

Fig. 1. General view of tribotesters: $a-2070$ SMT-1; b MAST-1

The tribological characteristics of rapeseed and mineral oils, as well as rapeseed oil based lubricants, during a multifactorial experiment to determine the optimal blending ratio of additives, were evaluated by the wear scar diameter of the bottom balls on a four-ball 
MAST-1 wear machine. The studies were carried out under the following conditions: the temperature of the test lubricant was $75^{\circ} \mathrm{C}$, the axial load was $200 \mathrm{~N}$, the rotation frequency of the top ball was $1500 \mathrm{~min}^{-1}$, and the duration of the experiment was $20 \mathrm{~min}$. The wear spot diameter was measured using an MIB-1 microscope with an eyepiece micrometer in two mutually perpendicular directions on each of the three fixed bottom balls. The three bottom balls were held firmly in a ball pot at an angle of $35^{\circ} 16^{\prime}$ to ensure a stable position of the wear scar to the optical axis of the microscope to exclude its visible distortion. Each experiment was repeated three times.

The lubrication blend was also evaluated using a 2070 SMT-1 wear machine during a multifactorial experiment. Special techniques were used to evaluate the effect of each type of additive and blending ratio, abrasive concentrations in the lubricating medium at the friction torque, time interval before the pre-seizure state of the bearings, mass wear of the bearings when the lubricant was applied.

To evaluate the extreme-pressure capacity, oil dosed in a volume of $2 \mathrm{~mm}^{3}$ was introduced into the contact zone and uniformly distributed over the friction surfaces of the ball bearings at low rotational speed without load. The main stage involved setting the required load and rotational speed, as well as recording the friction torque and temperature on a scale tape before a pre-seizure state to occur in the friction pair. The time of the pre-seizure state was recorded by a sharp increase in the friction torque between the balls.

During wear evaluation, to lubricate the friction pair the bottom ball was immersed to a depth of $5 \mathrm{~mm}$ in an oil bath with a volume of $170 \mathrm{~mm}^{3}$. Tests lasted 1 hour at a load of $2000 \mathrm{~N}$ and a rotational speed of $1000 \mathrm{~min}^{-1}$ with preliminary running-in for 15 minutes with a gradual increase in the loading. The test bearings were preliminarily weighed on a VLA-200M analytical balance with an accuracy of $0.2 \mathrm{mg}$. Upon completion of the test, the balls were thoroughly washed, dried, and reweighed, after which the wear amount was determined from the change in the mass of the samples.

Experimental samples of the test lubricants with a volume of $500 \mathrm{ml}$ were prepared to evaluate the effect of abrasive impurities in accordance with GOST 8002-62 on the anti-wear properties of rapeseed and mineral oils, as well as the lubrication formula. The blending ratio of abrasive particles $\left(\mathrm{S}=5600 \mathrm{~cm}^{2} / \mathrm{g}\right)$ in all cases remained constant and amounted to $0.25 \%$ of the weight of the sample. The required mass of abrasive dust $\left(\mathrm{m}_{\mathrm{a}}\right)$ for preparing a sample of oil at low levels of contamination was determined by the formula:

$$
m_{a}=0,01\left(\gamma_{a}-\gamma_{a 0}\right) V_{s} \rho_{o}, \mathrm{~g},
$$

where $\gamma_{a}$ is the required amount of abrasive impurities in the oil, $\% ; \gamma_{a 0}$ is the amount of abrasive impurities in fresh oil, $\% ; V_{s}$ is the volume of the experimental sample of oil, $\mathrm{mm}^{3} ; \rho_{o}$ is oil density, $\mathrm{g} / \mathrm{mm}^{3}$.

To ensure uniform distribution of abrasive particles throughout the lubricating medium, the experimental sample was heated to a temperature of $70-80^{\circ} \mathrm{C}$ and stirred for 1 hour.

\subsection{Multifactorial Experiment}

Based on the literature [11], anti-wear and antioxidant D-11, anti-wear, extreme pressure and anti-friction EFO, graphite (antifriction additive), and anti-foam PMS200A were selected for targeted additization of rapeseed oil. The blending ratio of additives for various functional purposes in a vegetable-mineral lubricant formula based on rapeseed oil was improved through a full factorial experiment $3^{2}$.

With the MAST-1 wear machine, the wear scar diameter of the balls was taken as the optimization parameter $\mathrm{Y}$, while the amount of the additive $\left(\mathrm{X}_{1}\right)$ and the amount of the abrasive $\left(\mathrm{X}_{2}\right)$, respectively, served as two independent factors. The experimental results were processed on a computer using the MathCad 14.0 and Statistica 6.0 programs. The intervals between the blending volume ratios and their values on a physical scale at the basic, top and bottom levels are presented in Table 3.

Table 3. Factors, intervals and levels of blending volume (for example, D-11).

\begin{tabular}{|c|c|c|}
\hline $\begin{array}{c}\text { Levels and } \\
\text { intervals of } \\
\text { blending ratio }\end{array}$ & $\begin{array}{c}|c| \\
\text { Fmount of D-1 } \\
\boldsymbol{X}_{\boldsymbol{1}}, \boldsymbol{\%}\end{array}$ & $\begin{array}{c}\text { Amount of } \\
\text { abrasive } \boldsymbol{X}_{\mathbf{2}}, \boldsymbol{\%}\end{array}$ \\
\cline { 2 - 3 } Basic level, $x_{i}=0$ & 3.0 & 0.125 \\
\hline $\begin{array}{c}\text { Interval of } \\
\text { blending ratio, } I_{i}\end{array}$ & 2.0 & 0.125 \\
\hline $\begin{array}{c}\text { Top level, } x_{i}=1 \\
\text { Bottom level, } x_{i}=- \\
1\end{array}$ & 5.0 & 0.25 \\
\hline
\end{tabular}

When all the requirements were met with a confidence level of $\alpha=0.95$, a regression equation was obtained:

$$
\begin{aligned}
& y=0.3387-0.0358 \cdot x_{1}+0.0283 \cdot x_{2}+ \\
& +0.0055 \cdot x_{1} \cdot x_{2}+0.0121 \cdot x_{1}^{2}+0.0032 \cdot x_{2}^{2}
\end{aligned}
$$

Attempts to improve the blending ratio of the additive during tests are characterized by the conditional extremum of the regression equation (2), that is, by determining the smallest value of the response in the range of values limited by the selected intervals of blending volume ratio. To find the minimum of regression equations, the built-in program "Minimize" of the mathematical program MathCad 14.0 was used.

To improve the extreme pressure and anti-friction properties, EFO and graphite additives were added to the lubricating blend. To improve the blending ratio of EFO and graphite additives the authors followed the same scenario performed to improve the D-11 additive. As a result, regression equations (3) and (4) were obtained, characterizing the effect on the optimization parameter 
of the blending ratios of abrasive, EFO and graphite additives, respectively.

$$
\begin{aligned}
& y=296.555+31.777 \cdot x_{1}+48.0 \cdot x_{2}+ \\
& +1.889 \cdot x_{1} \cdot x_{2}-10.470 \cdot x_{1}^{2}+1.085 \cdot x_{2}^{2} \\
& y=2.996-0.126 \cdot x_{1}+0.744 \cdot x_{2}+ \\
& +0.019 \cdot x_{1} \cdot x_{2}+0.030 \cdot x_{1}^{2}-0.011 \cdot x_{2}^{2}
\end{aligned}
$$

Upon completion of the process of optimizing the blending ratio of additives in the lubricating formula, comparative wear of mineral oils and lubricant was tested on the 2070 SMT-1 wear machine.

\section{Findings}

The comparative laboratory results of mineral and rapeseed oil on the MAST-1 and 2070 SMT-1 tribotesters are presented in Table 4.

Table 4. Comparative laboratory results of oils.

\begin{tabular}{|c|c|c|c|}
\hline Oil & $\begin{array}{c}\text { 2070 SMT-1 } \\
\text { Time to } \\
\text { seizure, } \mathbf{s}\end{array}$ & $\begin{array}{c}\text { 2070 SMT-1 } \\
\text { Friction } \\
\text { torque, } \text { Nm }\end{array}$ & $\begin{array}{c}\text { MAST-1 } \\
\text { Wear scar } \\
\text { D, mm }\end{array}$ \\
\hline $\begin{array}{c}\text { Rapeseed } \\
\text { oil }\end{array}$ & 170 & 4.1 & 0.420 \\
\hline M-10 G 2 & 281 & 3.5 & 0.290 \\
\hline $\begin{array}{c}\text { MGE- } \\
\text { 46V }\end{array}$ & 210 & 3.7 & 0.310 \\
\hline
\end{tabular}

The results show that with rapeseed oil, the wear scar diameter is $26.2 \%$ and $31 \%$ larger than that of mineral oils MGE-46V and $\mathrm{M}-10 \mathrm{G}_{2}$, respectively. Besides, rapeseed oil has less time to seizure by $39.5 \%$ and $19 \%$ than $\mathrm{M}-10 \mathrm{G}_{2}$ and MGE- $46 \mathrm{~V}$, respectively; mass wear of samples is $8.8 \%$ and $5.0 \%$ higher than that of $\mathrm{M}-10 \mathrm{G}_{2}$ and MGE-46V, respectively; friction torque is more by $14.6 \%$ and $9.8 \%$ than that of $\mathrm{M}-10 \mathrm{G}_{2}$ and MGE-46V, respectively. Consequently, the use of pure rapeseed oil in hydraulic systems of agricultural vehicles is impossible due to its low tribological performance. Thus, additizing rapeseed oil to obtain a lubricant blend is a relevant issue.

The results of a full factorial design to optimize the blending ratio of additives in the lubricant are shown in Figure 2.

The response surface based on the results of a full factorial design (Fig. 2, a) made it possible to establish that the minimum response value (wear scar diameter) occurred at $3.7 \%$ D-11 additive blend. The resulting formula (rapeseed oil $+3.7 \%$ D-11) obtained on the 2070 SMT-1 wear machine to determine the time to seizure and the friction torque showed that these parameters have a value equal to $205 \mathrm{~s}$ and $3.8 \mathrm{Nm}$, respectively. A comparative analysis showed that the time to seizure in the lubricating blend was $20.6 \%$ longer than that of rapeseed oil, but $37 \%$ less than that of M$10 \mathrm{G}_{2}$ oil, while the friction torque of the lubricating blend is $7.3 \%$ less than that of rapeseed oil and $8.6 \%$ more than $\mathrm{M}-10 \mathrm{G}_{2}$ oil.

The maximum of the regression equation (3) characterizes the optimal EFO amount (Fig. 2, b) according to the maximum time to seizure. EFO additive being introduced at a concentration of $3.2 \%$ into the lubricating formula increased the seizure time to $341 \mathrm{~s}$, which is twice higher than that of rapeseed oil and $21.3 \%$ higher than that of $\mathrm{M}-10 \mathrm{G}_{2}$ oil. Moreover, the torque value decreased, insignificantly though, and amounted to $3.7 \mathrm{Nm}$.

a)

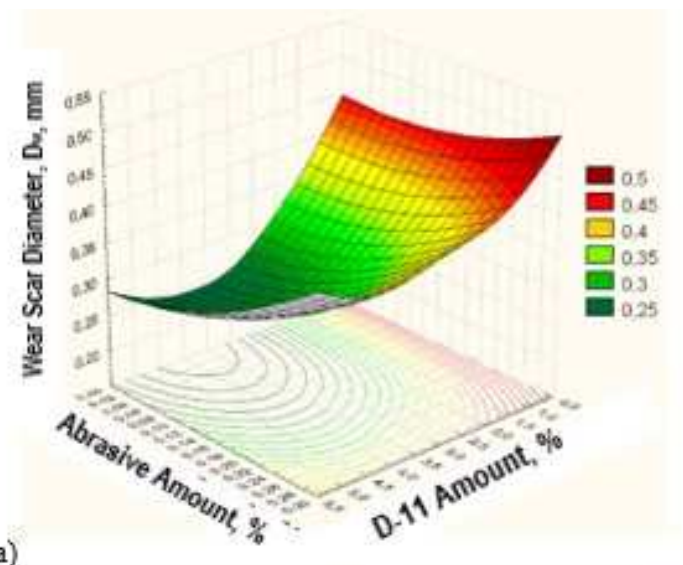

b)
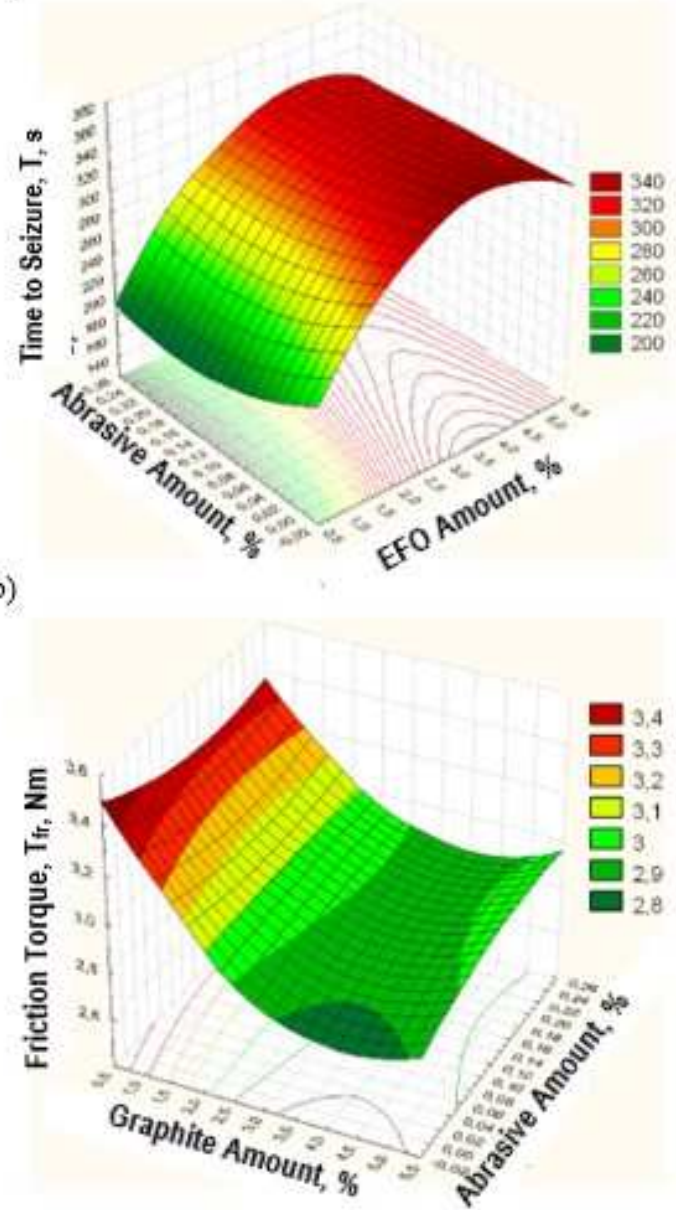

c)

Fig. 2. Response surfaces: a) optimization of D-11 amount; b) optimization of EFO amount; c) optimization of graphite amount

The minimum of the regression equation (4) characterizes the optimal concentration of graphite (Fig. 2 , c) according to the minimum torque. The minimum friction torque of $2.8 \mathrm{Nm}$ is achieved at a graphite blend of $4.197 \%$. 
Thus, the blended lubricant "88.9\% rapeseed oil + $3.7 \%$ D-11 $+3.2 \% \mathrm{EFO}+4.197 \%$ graphite $+0.003 \%$ PMS-200A" is recognized as optimal.

At the final stage, wear of mineral oils and the obtained rapeseed oil based blend were compared on the SMT-1 wear machine (Fig. 3).

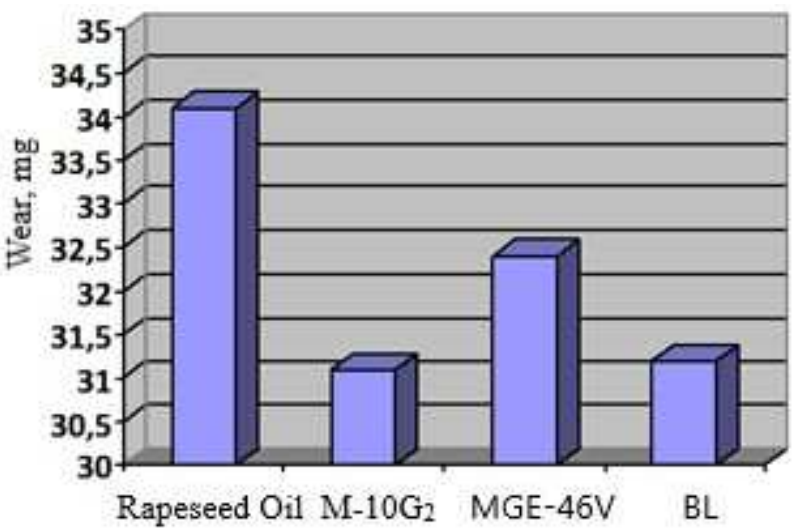

Fig. 3. Wear of samples

Based on the results of the factorial experiment, a lubricating formula containing $88.9 \%$ rapeseed oil, $3.7 \%$ anti-wear and antioxidant additive D-11, 3.2\% anti-wear, extreme pressure and anti-friction additive EFO, 4.197\% graphite (antifriction additive) and $0.003 \%$ anti-foam additive PMS-200A, has more advanced tribological characteristics as compared to MGE-46V hydraulic oil, but is somewhat inferior to $\mathrm{M}-10 \mathrm{G}_{2}$ oil. Accordingly, the blended lubricant is recommended for use as a working fluid in hydraulic systems of agricultural vehicles.

\section{Conclusion}

The graphical dependences obtained experimentally indicate that rapeseed oil is inferior in tribological properties to mineral oils and requires targeted additization for use as a working fluid in hydraulic systems of agricultural vehicles. Further studies aimed at improving the lubricating blend showed that it is advisable to add $3.7 \%$ D-11 additive, $3.2 \%$ EFO additive, $0.003 \%$ PMS-200A additive, and $4.197 \%$ colloidal graphite to the base rapeseed oil. This lubricating blend is superior in tribological properties to the mineral hydraulic oil MGE-46V recommended for use in hydraulic systems of agricultural vehicles.

\section{References}

1. A. I. Petrashev, V. V. Klepikov, Influence of oil leaks on the operation of hydraulic machines, Agricultural equipment: maintenance and repair, 6, 11-17 (2020)

2. V. A. Dovgyalo, V. L. Moiseenko, K. V. Maksimchik, N. V. Pismennaya, Special selfpropelled rolling stock. Research of the need and development of consumption rates for lubricants for operation and repair, Mining mechanics and mechanical engineering, 4, 70-78 (2020)

3. V.E. Olishevskaya, G.S. Olishevsky, Comparative analysis of technologies for the regeneration of automotive lubricants, Contemporary Innovation Technique of the Engineering Personnel Training for the Mining and Transport Industry Modern, 1(5), 180-192 (2018)

4. L.A. Chernyshev, T.A. Startseva, D. Yu. Mikhailenko, Ecological problem of waste motor oils, Eco-potential, 2(22), 91-95 (2018)

5. I. Y. Hwang, A. D. Nguyen, T. T. Nguyen, L. T. Nguyen, O. K. Lee, E. Y. Lee, Applied microbiology and biotechnology, 102, 3071 (2018)

6. N. Parsaeifard, M. Sattler, B. Nasirian, V. C. P. Chen, Waste management, 106, 44 (2020)

7. D. Woo, T. Kim, Journal of Mechanical Science and Technology, 33, 3655 (2019)

8. M. H. Jabal, R. Abdulmunem, S. Hussain, Journal of the Air \& Waste Management Association, 69, 109 (2019)

9. E. Wozniak, E. Waszkowska, T. Zimny, S. Sowa, T. Twardowski, Frontiers in plant science, 10, 1423 (2019)

10. I.G. Kazarova, Plant growing as a leading branch of the farming industry of Russia, Modern research and development, 3(4((21)), 232-234 (2018)

11. O.S. Volodko, A.P. Bychenin, D.N. Bazhutov, Vegetable-mineral lubricant composition for hydraulic systems, Farm Machinery Operator, 6, 3031 (2019) 